\title{
Magnetic and electric Aharonov--Bohm effects in nanostructures
}

\author{
I.O. Kulik' \\ Department of Physics, Bilkent University, 06533 Bilkent, Ankara, Turkey
}

\begin{abstract}
The paper reviews and extends the magnetic Aharonov-Bohm effect (persistent current, resistance oscillation) in normalmetal rings including spin-independent and spin-dependent hopping, Zeeman splitting, magnetic textures and wheels, ring rotation and weak coupling, as well as the electric Aharonov-Bohm effect ("persistent charge") in small metallic contacts. We then discuss dynamical screening effects in a surface charge in a metal. Energy dissipation due to motion of the surface charge has a singularity at the velocity of motion equal to the phonon propagation velocity. Surface image of an external charge inside the metal is strongly distorted at the velocity of motion larger than the Fermi velocity.
\end{abstract}

\section{Introduction}

Small size conductors develop a number of peculiar effects at low temperature. These include ballistic electron transport through point contacts, surface charge accumulation, quantization of electron charge and magnetic flux in small metallic loops. The subject of this paper is related to Aharonov-Bohm effects in solids [1], the role played by magnetic vector potential (the "magnetic" effect) and by electrostatic scalar potential ("electric" Aharonov-Bohm effect), as well as to consideration of dynamical effects associated with the Thomas-Fermi screening of a surface charge (the "surface spectroscopy").

Magnetic Aharonov-Bohm effect manifests itself in the oscillation of electrical resistance of nanoscale loop or network as a function of applied magnetic flux $\Phi_{\mathrm{M}}$, and in the appearance of a persistent current in a closed-loop oscillating versus flux with the period $\Phi_{0}=h c / e[2,3]$. Disorder and inelastic scattering reduces the magnitude of persistent current. A detailed shape of $J\left(\Phi_{\mathrm{M}}\right)$ dependence in a mesoscopic structure permits pattern recognition from a mesoscopic "fingerprint" because cusps in $J\left(\Phi_{M}\right)$ are related to broken links in a network.

\footnotetext{
${ }^{1}$ On leave of absence from B.Verkin Institute for Low Temperature Physics and Engineering, Acad. Sci. of Ukraine, 47 Lenin av., 310164 Kharkov, Ukraine.
}

Rotation of a loop results in the appearance of a persistent current proportional to angular velocity of rotation $\omega$, at zero magnetic field. The electric Aharonov-Bohm effect is a counterpart of magnetic Aharonov-Bohm effect related to the "electric flux" $\Phi_{\mathrm{E}}=c \int E \mathrm{~d} x \mathrm{~d} t$. Tunneling or point contact placed in an electric field, swept with period $T$, will develop electric polarization periodic in $\Phi_{\mathrm{E}}$ with period $\Phi_{0}$. Another manifestation of the effect is in the oscillation of contact capacitance versus $\Phi_{\mathrm{E}}$.

The paper also includes a discussion of non-quantummechanical effects associated with surface charges, or edge electronic states, in metals. Surface charge motion in a metal results in a specific energy loss mechanism. For the electronically driven motion of a surface charge with velocity $V$, the loss will have pronounced singularity at the Fermi velocity $v_{\mathrm{F}}$ due to the crisis of the Thomas-Fermi screening at $v_{\mathrm{F}}$, thus providing for the determination of Fermi velocity. In a cylindrically shaped or disk geometry, magnetic moment of a surface current will also have a singularity at the velocity of a driven motion at $V=v_{\mathrm{F}}$.

\section{Magnetic Aharonov-Bohm effect}

The electron states in the conductors of multiple connected geometry (rings, networks, etc.) are quantized with a magnetic flux serving as a control parameter for discrete 
energies. As a result, the total energy $E$ of a system becomes flux-dependent resulting in the appearance of a persistent current $\left(\Phi=\Phi_{M}\right)$

$J=-c \hat{\partial} E / \partial \Phi$,

which is a stable current in the equilibrium state, nondecaying in time for arbitrary long interval; nevertheless, the electron scattering and energy dissipation are taken into account. Such current was first calculated in a metal with long mean free path in Ref. [2] (and with arbitrary mean free path in Ref. [3]). The opposite case of short mean free path $l \ll L(L=2 \pi R$ is ring circumference) was considered by Altshuler et al. [4] in the context of disorder-induced electron interference phenomena in the conductivity of metals ("weak localization"). The latter effect manifests itself in the resistance oscillation with a twice smaller period, $\Phi_{1}=\Phi_{0} / 2$, and does not show up in the thermodynamics. Both types of oscillation exist provided sample size is smaller than the characteristic length $l_{\varphi}=v_{\mathrm{F}} \tau_{\varphi}$ where $\tau_{\varphi}$ is the phase-breaking time ( $\tau_{\varphi}=\tau_{\varepsilon}$ in the ballistic regime $l \gg L$ and $\tau_{\varphi}=\sqrt{\tau \tau_{c}}$ in the diffusive regime $\left.l \ll L\right)$. $\tau=l / v_{\mathrm{F}}$ is the elastic scattering time and $\tau_{\varepsilon}$ the inelastic scattering time determined by electron-phonon interaction $\tau_{=}^{\text {ep }} \sim \hbar \theta_{\mathrm{D}}^{2} / k_{\mathrm{B}} T^{3}$ and (specifically at temperatures below $1 \mathrm{~K}$ ) by electron-electron interaction $\tau_{\Xi}^{\mathrm{ee}} \sim \hbar \varepsilon_{\mathrm{F}} / k_{\mathrm{B}}^{2} T^{2}$.

The Hamiltonian of an electron in the ring in the tightbinding approximation is

$$
\begin{aligned}
& H=-\sum_{n \times \beta} a_{n \times}^{-}\left[t_{0}+t_{1}\left(\mathrm{e}^{\mathrm{i} i} \sigma_{-}-\mathrm{e}^{-\mathrm{i}_{i j}} \sigma_{-}\right)\right]_{x \beta} a_{n+l, \beta} \mathrm{e}^{\mathrm{i} \theta} \\
& + \text { h.c. }+\sum_{n \times \beta} a_{n \alpha}^{+}\left[V\left(\varphi_{n}\right)-\mu B_{z} \sigma_{z}-\mathrm{e}^{-\mathrm{i} \varphi_{t \prime}} \mu\left(B_{r}-\mathrm{i} B_{\varphi}\right) \sigma_{+}\right. \\
& \left.-\mathrm{e}^{\mathrm{i} \varphi_{i j}} \mu\left(B_{r}+\mathrm{i} B_{\varphi}\right) \sigma_{-}\right]_{x \beta} a_{n j}
\end{aligned}
$$

with arbitrary $;$ and real $t_{0}, t_{1} . \theta=2 \pi \Phi / N \Phi_{0}$ where $N$ is the number of sites in the loop, $\varphi$ is the angular coordinate along the loop, and $\sigma_{ \pm}=\left(\sigma_{x} \pm \mathrm{i} \sigma_{y}\right) / 2 \cdot a_{n x}^{+}$is an operator creating electron at site $n$ with spin projection $\alpha$. The Hamiltonian (2) includes spin-independent and spin-dependent (spin-orbit coupled) hopping between sites interacting with magnetic flux due to the electron charge $e$, and to the external magnetic field $\boldsymbol{B}$ due to the electron magnetic moment $\mu$. The effect of radial magnetic field $B$, ("magnetic texture") was considered by Loss et al. [5] and that of the azimuthal magnetic field $B_{\varphi}$ ("magnetic wheel") by Stern [6].

$V(\varphi)$ is a potential representing disorder in the ring. $\mathrm{Ne}$ glecting the latter and the Zeeman coupling terms in Eq. (2), we obtain the electron energy independent of $\gamma$ :

$$
\begin{aligned}
& E_{m 1}=-2 t \cos \left[\frac{2 \pi}{N}(m+v) \pm \gamma_{1}\right], \quad t=\sqrt{t_{0}^{2}+t_{1}^{2}}, \\
& \gamma_{1}=\arctan \frac{t_{1}}{t_{0}}
\end{aligned}
$$

where $m$ is an integer and $v=\Phi / \Phi_{0}$.
The energy versus flux dependence is shown in Fig. 1. The spin-orbit coupling splits minimum of $E(\Phi)$ but does not change the position of the maximum in the lowest-energy state. This results in the appearance of strong second harmonics of $E(\Phi)$ and can eventually lead to halving of oscillation period from $\Phi_{0}$ to $\Phi_{0} / 2$ [7].

If we neglect the spin-orbit effects in Eq. (2), energy can be obtained in an exact form,

$$
\begin{aligned}
E_{m}= & \frac{1}{2}\left(\varepsilon_{m \uparrow}+\varepsilon_{m+1, \downarrow}\right) \\
\pm & {\left[\frac{1}{4}\left(\varepsilon_{m \uparrow}-\varepsilon_{m-1, \downarrow}\right)^{2}+\mu^{2}\left(B_{r}^{2}+B_{\varphi}^{2}\right)\right]^{1 / 2}, } \\
\varepsilon_{m \uparrow, m \downarrow} & =-2 t_{0} \cos \frac{2 \pi(m+v)}{N} \mp \mu B_{z},
\end{aligned}
$$

shown in Fig. 1. It is interesting to note that radial and azimuthal fields have effect seemingly similar to that of the Aharonov-Bohm flux (in particular, current does not vanish at $\Phi=0$, see right panel of Fig. 1). This however does not produce a nonzero total persistent current (contrary to the statement in Ref. [5]) because, due to time-reversal symmetry, states $m$ and $-m+1$ at $\Phi=0$ correspond to opposite currents and cancel each other after averaging over the electron distribution.

A disorder in the system reduces the magnitude of the persistent current. For a one-dimensional ring, average current can be estimated as

$J \sim \frac{e v_{\mathrm{F}}}{R} \mathrm{e}^{-2 \pi R / \xi} \mathrm{e}^{-2 \pi R / \check{s}} \sin 2 \pi \frac{\Phi}{\Phi_{0}}$

with $\xi=4 l$ and $\zeta=\hbar v_{\mathrm{F}} / 2 \pi T$. Currents of such order of magnitude have been observed in the experiment $[8,9]$.

For a multichannel ring with a number of perpendicular conducting channels $N_{\perp}=k_{\mathrm{F}}^{2} \Sigma / 4 \pi$ ( $\Sigma$ is the cross-section of a ring), coherence length $\xi$ increases proportional to the number of channels $\xi \sim l N_{\perp}$ [11]. In the metallic diffusive regime $l \ll L \ll \xi$ appropriate to the experiment, the amplitude of a preexponential factor in Eq. (5) is of the order of $l / L[12,13]$. The magnitude of a current observed in Ref. [8] is much larger than this value and therefore hard to interpret on the basis of current understanding of quantum transport in disordered conductors. The $\Phi_{0} / 2$-periodic current has been calculated in Ref. [14] and observed in
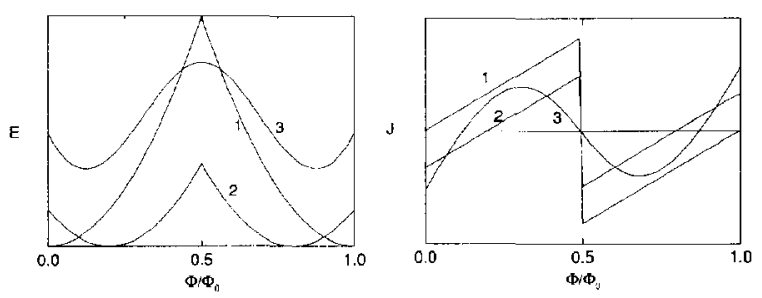

Fig. 1. Left panel: energy of a ring versus magnetic flux. Right panel: persistent current versus flux. (1) $\gamma=0, B=0$; (2) $\gamma=0.2, B=0 ;$ (3) $\gamma=0, B_{z}=0, \mu B_{\perp}=\frac{1}{3} \varepsilon_{0}$. 
the experiment [15]. The observation of such periodicity is simpler than the observation of $\Phi_{0}$-periodic persistent current because it can be done in an ensemble of large number ( $\sim 10^{7}$ in Ref. [15]) of rings, whereas $\Phi_{0}$-periodic currents are random in magnitude and sign and therefore cancel each other in the large ensemble.

In a one-dimensional ring, potential $V$ influences persistent current not in exactly the same way as it changes the conductance of the ring [10]. For a single barrier in the ring, $V(\varphi)=V \delta(\varphi)$, current is easily calculated:

$J_{m}=(-1)^{m} \frac{e m^{2}}{2 \pi V} \sin 2 \pi \frac{\Phi}{\Phi_{0}}$

and is proportional to square root of the barrier transmissivity $D$ rather than to $D\left(D \sim 1 / V^{2}\right)$. Therefore, the persistent current in a "weakly coupled" mesoscopic ring scales with $R^{-1 / 2}$ rather than with $R^{-1}$ where $R$ is the ring resistance. (We mention that the ring, if superconducting, has critical current proportional to $R^{-1}$ which therefore decreases more rapidly with $R$ than the persistent current; the former is however much larger than the latter because it shows up as the effect of a single electron, see Eq. (5), rather than the effect of macroscopic number of electrons.)

If mesoscopic ring is rotating with the angular velocity $(s)$ along its axis of symmetry, persistent current will be generated without the external magnetic flux. Rotation is equivalent to the effective vector potential

$A=\frac{m c}{e} \omega R$

which produces the phase difference $\theta=2 \pi e A R / N \hbar c$ between the sites in Eq. (2). An estimate of the induced magnetic moment of the ring is

$\frac{M^{\prime}}{\mu_{\mathrm{B}}} \sim \frac{m^{2} v_{\mathrm{F}}}{\hbar^{2}} \omega R^{3}$

where $\mu_{\mathrm{B}}$ is the Bohr magneton. This moment is too small to be observed in a single ring. Consider however the crystal comprising of large organic molecules like e.g. $\mathrm{C}_{60}$. Assuming that the number of molecules per unit volume $n \sim R^{-3}$, we obtain for the magnetic field produced by persistent currents,

$H^{\prime} \sim \frac{e^{2}}{\hbar c} \frac{v_{\mathrm{F}}}{c} H_{0,}, \quad H_{1,}=\frac{2 m c}{e}(\omega)$

( $H_{i,}$ is a reference field of a London moment [16] induced by the rotation of a superconductor).

The field (9) is of order $H^{\prime} \sim 10^{-11} \mathrm{G}$ per revolution per second, and can in principle be detected in an experiment. We mention that the requirement that ring is metallic is not strictly necessary because as was shown in Ref. [17], persistent current in principle can exist in insulators.
A levitating metallic ring (say, ring floating over the surface of liquid helium supported by surface tension) can exhibit much more delicate quantum effects. Because the ring is comprised of positive ions interacting with the external flux, the Aharonov-Bohm arguments concerning the role played by vector potential in quantum mechanics, equally apply to both electronic and ionic subsystems. Assuming that ions are immobile in the frame of reference of the ring, we conclude that their response to flux will be that of a charge $-\mathrm{Ne}$ ( $N$ is total number of ions equal to the number of electrons ). As a result, the flux quantum corresponding to the lattice will be $\Phi_{0} / N$ rather than $\Phi_{0}$. Such effect requires much stronger restriction on the crystal purity and temperature than the electronic Aharonov-Bohm effect. Fractional Aharonoy-Bohm effect was also predicted [18] for the immobile ring, as a consequence of strong electron-electron correlation.

In the above discussion, we considered electronic system in equilibrium. This requires that inelastic relaxation time is not too long to allow electrons to follow adiabatically after slowly changing the magnetic flux. Specific relaxation of electrons, that of inelastic electron backscattering [19], is operative in establishing the equilibrium between the electrons. If the corresponding relaxation time is too long, persistent current might change, or vanish.

\section{Electric Aharonov-Bohm effect}

The change of the electron phase due to electrostatic potential has not been so far detected in any experiment (the electric-field-induced shift in the magnetic Aharonov-Bohm oscillation observed in Ref. [20] is not related to this effect and will be discussed elsewhere). To clarify the requirement for the observation of the electric Aharonov-Bohm effect in solids, consider Fig. 2. Two metallic particles are placed above and below a thin capacitor with the electric field $\boldsymbol{E}$ confined within the capacitor. The effect of $\boldsymbol{E}$ on the tunneling between the particles will in no way be changed when the distance between the plates of the capacitor increases, provided the potential between particles remains unchanged. This is also true when plates of the capacitor are outside the particles (Fig. 2(c)). Assuming that potential $V(t)$ is periodic in time with a period $T_{0}$, introduce electric flux as $\Phi_{\mathrm{E}}=$ $c \int_{0}^{T_{0}} V(t) \mathrm{d} t$. The problem is reduced to that of a two-level system with a Hamiltonian

$H=\frac{e V}{2} \sigma_{z}+T_{12} \sigma_{x}$,

where $T_{12}$ is the transfer matrix element between the levels.

For $V(t)$ in the form of a periodic Kronig-Penney potential

$V(t)=V_{0} \sum_{n} \delta\left(t-n T_{0}\right)$ 
time evolution of a two-component wave function $\Psi=$ $(u, v)$ is easily found giving nonzero dipole momentum $P=$ $e\left(|u|^{2}-|v|^{2}\right)$

$P(t)=4 e T_{12} T \operatorname{Re}\left(u_{0}^{*} v_{0}\right) \operatorname{Im} \zeta(t)$,

where

$\zeta(t)=\frac{\mathrm{e}^{2 \pi \mathrm{ir}}-\mathrm{e}^{2 \pi \mathrm{iNr}}}{1-\mathrm{e}^{2 \pi \mathrm{ir}}}+F \mathrm{e}^{2 \pi \mathrm{iNr} r}, \quad v=\frac{e V_{0}}{h}$,

$N=\left[\frac{t}{T_{0}}\right], \quad F=\left\{\frac{t}{T_{0}}\right\}$,

$[x]$ and $\{x\}$ are integer and fractional parts of $x$, respectively. $v$ in Eq. (13) is the ratio of electrical flux to flux quantum, $v^{\prime}=\Phi_{\mathrm{E}} / \Phi_{0} . P(t)$ is proportional to the first order of the tunneling amplitude $T_{12}$, similar to the first-order tunneling current in a weakly coupled mesoscopic ring (6).

Assume that $\Phi_{\mathrm{E}}$ slowly changes in time and average (13) with respect to fast oscillation, and with respect to random variation of $\Phi_{\mathrm{E}}$, with a characteristic Gaussian width $\gamma=$ $\Delta \Phi_{\mathrm{E}} / \Phi_{0}$. This gives at $T=0$,

$\bar{P}(t)=\sum_{s=1}^{\infty}(-1)^{s} A_{s} \mathrm{e}^{-\pi^{2} s^{2} y^{2}} \sin \left(2 \pi s \frac{\Phi_{\mathrm{E}}}{\Phi_{0}}\right)$.

The effective charge difference between particles oscillates as a function of the average electric flux $\Phi_{\mathrm{E}}$ with the period of a flux quantum $\Phi_{0}$. The amplitude of the sth harmonics of the oscillation at zero temperature equals

$A_{s}=\frac{4}{\pi} \int_{0}^{\pi / 2} \cot x \sin (2 s x) \mathrm{d} x$

The effect of such oscillation can only be observed for a time interval smaller than the inelastic relaxation time $\tau_{\varepsilon}$ at temperature smaller than $\hbar / T_{0}$. It is also required that the voltage be larger than $V_{C}=e^{2} / C$ to eliminate the effect of
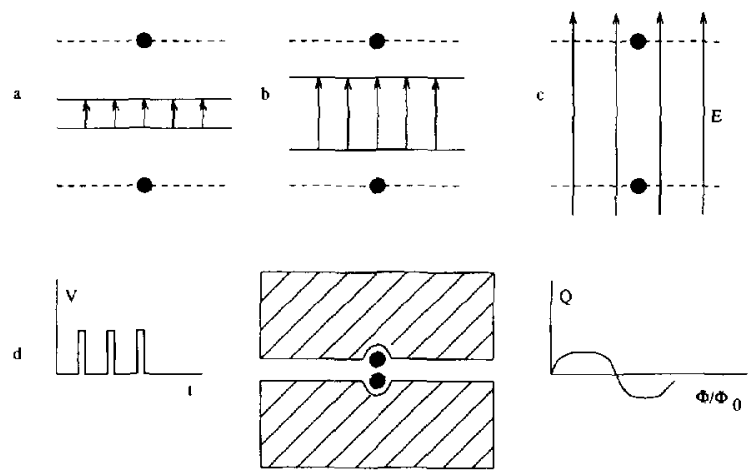

Fig. 2. Gedankenexperiment of the Aharonov-Bohm effect in a tunneling junction with capacitor inside $(a, b)$ and outside $(c)$ of the junction. (d) Schematic of an experiment intended to search for the persistent charge. a Coulomb blockade in small tunneling junctions (e.g., Ref. [21]). The latter effect can be reduced if metallic dots are placed near the bulk metallic electrodes, as schematized in Fig. 2(d).

\section{Surface spectroscopy of conduction electrons}

Consider metallic semispace in the near vicinity of an electrode (a tip) biased, say, positively with respect to the surface (Fig. 3). The electron charge is induced inside a metal near its surface making a surface image of a tip. We now investigate dynamical screening effects associated with the motion of tip along the metal surface.

The Fourier harmonics $\phi_{k}=\phi$ of an electrostatic potential inside a metal satisfies the equation

$k^{2} \phi-\frac{\mathrm{d}^{2} \phi}{\mathrm{d} z^{2}}=-4 \pi e N\left(\varepsilon_{\mathrm{F}}\right)\langle\chi\rangle$,

where $\chi$ is the correction to the electron distribution function

$f_{\mathbf{p}}=f_{0}+\chi(x-V t) \frac{\partial f_{0}}{\hat{\partial} \varepsilon}$.

By Poisson transformation, $\phi$ is represented in the form

$\phi(z)=\frac{1}{2 \pi \mathrm{i}} \int_{a-i \infty}^{a+\mathrm{i} \infty} \mathrm{d} p \mathrm{e}^{p z} \frac{p \phi(0)+\phi^{\prime}(0)+\int_{-\pi}^{\pi} \frac{\mathrm{d} \varphi}{2 \pi} \frac{A_{u}}{p+\mathrm{i}_{i, p}}}{p^{2}-k^{2}-1-S(p)}$

with

$$
S(p)=\int_{-\pi}^{\pi} \frac{\mathrm{d} \varphi}{2 \pi} \frac{k V}{\left(\gamma_{\varphi}(p)-i p\right) \sin \varphi}, \quad \gamma_{\varphi}=\frac{k(\cos \varphi-V)}{\sin \varphi} .
$$

$\varphi$ is azimuthal coordinate at the cylindrical Fermi surface (we use dimensionless units such that $v_{\mathrm{F}}=1$ and $N\left(\varepsilon_{\mathrm{F}}\right)=$ 1). The boundary condition for the electrostatic potential at the metal surface takes the form ( $p_{0}$ is the pole of denominator of Eq. (18))

$\phi^{\prime}(0)=-p_{0} \phi(0)-\int_{0}^{\pi} \frac{\mathrm{d} \varphi}{2 \pi} \frac{A_{\varphi}}{p_{0}+\mathrm{i}_{\gamma \varphi}}$,

where the prime denotes the derivative with respect to $z$, and $A_{\varphi}$ is a function satisfying an integral equation

$$
\begin{aligned}
\hat{L} A_{\varphi} & +\frac{q}{2} \frac{k V}{\sin \varphi} \int_{0}^{\pi} \frac{\mathrm{d} \varphi \rho^{\prime}}{2 \pi} \frac{1}{p_{0}^{2}+\gamma_{i^{\prime} \varphi}^{2}}\left(\frac{A_{\varphi \varphi}}{\gamma_{\varphi \varphi}+\gamma_{\varphi \rho}}+\frac{\hat{L} A_{\varphi \varphi}}{\gamma_{\varphi}-\gamma_{\varphi \rho}}\right) \\
& =\frac{k V}{\sin \varphi} \frac{\phi_{0}}{\gamma_{\varphi}-i p_{0}}, \quad \phi_{0}=\phi(0)+\int_{-\pi}^{\pi} \frac{d \varphi}{2 \pi} \frac{A_{\varphi}}{p_{0}^{2}+\gamma_{\varphi}^{2}},
\end{aligned}
$$


where $q$ is the coefficient of diffuse reflection of electron at metal surface and

$\hat{L} A_{\varphi}=(1-q) A_{\varphi}+\int_{0}^{\pi} A_{\varphi} \sin \varphi \mathrm{d} \varphi$.

The analysis of the above formulas shows that dynamical surface sheet of a metal has the same thickness $\lambda_{\mathrm{TF}}=$ $\left(4 \pi e^{2} N\left(\varepsilon_{\mathrm{F}}\right)\right)^{-1 / 2}$ as in the case of static Thomas-Fermi screening if the velocity is smaller than the Fermi velocity $v_{\mathrm{F}}$. At $V>v_{\mathrm{F}}$, there appears a component of $\phi$ penetrating inside the metal at a distance $k^{-1}$ of the order of surface-tip distance (or tip size). Such anomalous penetration vanishes if the corresponding potential component $\phi_{k}$ is equal to zero. Self-consistent solution for the potential distribution inside and outside the metal shows that the potential outside the metal will be substantially distorted compared to the static potential distribution. For a cylindrical Fermi surface, surface image elongates in the direction of the tip motion, as shown schematically in Fig. 3.

The energy dissipation related to the tip motion can be calculated from the boundary condition at the surface (22). For a spherical Fermi surface, we obtain an expression for the dissipated energy

$$
\begin{aligned}
W= & \frac{V^{2}}{4 \pi e^{2} N\left(\varepsilon_{\mathrm{F}}\right) v_{\mathrm{F}}} \frac{1-q / 2}{q} \int \frac{\mathrm{d}^{2} k}{(2 \pi)^{2}} k_{x}^{2}\left|\phi_{k}^{\prime}(0)\right|^{2} \\
& \times\left(\ln \frac{C_{1}}{\left|k_{x}\right| \lambda_{\mathrm{TF}}}+\eta \ln \frac{C_{2}}{\left|k_{\mathrm{r}}\right| i_{\mathrm{TF}}}\right),
\end{aligned}
$$

where $C_{1}$ and $C_{2}$ are constants of order of unity and $\eta$ is $V$-dependent quantity showing large increase as a function of velocity between $v_{\mathrm{F}}$ and critical velocity $V_{\mathrm{c}}$ satisfying the relation

$$
\frac{V_{\mathrm{c}}}{v_{\mathrm{F}}}=\tanh \frac{v_{\mathrm{F}}}{V_{\mathrm{c}}}, \quad V_{\mathrm{c}}=0.8335 v_{\mathrm{F}} .
$$

Above $V_{c}, W$ has singularity as a function of velocity. At larger velocity, linear regime of screening breaks down, and surface layer becomes reconstructed. This will be investigated elsewhere [24].

The coefficient of diffuse reflection is expected to be dependent on the electron energy. If the velocity of motion is

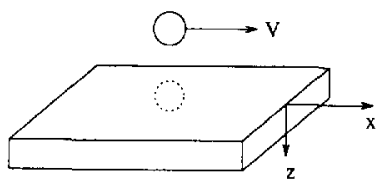

$\mathrm{V}<\mathrm{V}_{\mathbf{F}}$

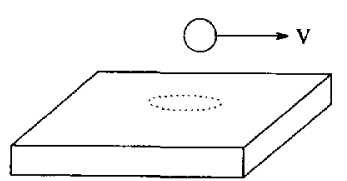

$\mathrm{V}>\mathrm{V}_{\mathrm{F}}$
Fig. 3. Surface image of a moving tip at the velocity of motion smaller and larger than the Fermi velocity. The axis of cylindrical Fermi surface is directed parallel to metal surface perpendicular to the direction of tip motion. equal to the phonon propagation velocity $s, q$ in Eq. (23) will increase due to the allowance for the surface phonon emission. Therefore, we may expect that the dependence $W(V)$ will have singularity near the phonon velocity similar to that in the conventional point-contact spectroscopy [22, 23].

Unlike in conventional conductivity measurements in metals in which the drift velocity of electron is extremely small, the velocity of surface sheet motion $V$ can in principle be made arbitrarily large in the linear regime (small $\phi)$. It is supposed that large values of $V$ can be achieved by propagating either charged particles or small charged bodies parallel to the metal surface. Another possibility is that charged solitons move in the semiconducting film overlaying (but not in direct electric contact with the metal surface), e.g. in the Gunn effect. Velocities of the Gunn domain motion in GaAs can be of the order of $10^{7} \mathrm{~cm} / \mathrm{s}$ (e.g., Ref. [25]).

\section{Conclusion}

The Magnetic Aharonov-Bohm effect is now a wellestablished phenomenon for systems with spatial periodicity of order $10^{3} \AA$ (nanostructures). The oscillating $h c / e$-periodic behavior in the conductivity of such structures is perfectly understood both theoretically and on experimental basis. However, the thermodynamics of small systems in the presence of the AB flux is less clear. Persistent current, an equilibrium lossless current in the ground state of normal metal, decreases dramatically in its magnitude with the increasing disorder and is exponentially small in non-metallic or insulating regimes. Experimental values of the current have unexpectedly proved to be larger than that based on theoretical calculation appropriate for a disordered metal. The study of persistent currents in disordered metallic systems represents the most direct way of testing the localization theories.

The electric Aharonov-Bohm effect has so far never been observed in an experiment. We have addressed this issue and showed the condition at which it shows up in a nanoscale metallic system. The hallmark of the effect may be analogous to the hc/e-periodic oscillation on magnetic flux, in the $h c / e$-periodic variation, as a function of electric flux of the "persistent charge" (and corresponding dipole moment) in double-well metallic systems.

Strongly correlated electronic models show $h c / e$ and $h c / 2 e$ periodicity in the dependence of their thermodynamic potential and the persistent current versus magnetic flux. The second effect is similar to superconductivity but not like the latter decreases in amplitude with the increasing system size. Mesoscopic (random in sign) and sign-conserving behavior of the current in the ring can be considered as a 
criterion for distinguishing between strongly correlated models proposed e.g. for the explanation of hightemperature superconductivity.

\section{References}

[1] Y. Aharonov and D. Bohm, Phys. Rev. 115 (1959) 485.

[2] I.O. Kulik, Pis'ma Zh. Eksp. Teor. Fiz. 11 (1970) 407 [JETP Lett. 11 (1970) 275].

[3] M. Buttiker, Y. Imry and R. Landauer, Phys. Lett. A 96 (1983) 365 .

[4] B.L. Altshuler, A.G. Aronov and B.Z. Spivak, Pis'ma Zh. Eksp. Teor. Fiz. 33 (1981) 101 [JETP Lett. 33 (1981) 94].

[5] D. Loss, P. Goldbart and A.V. Balatsky, Phys. Rev. Lett. 65 (1990) 1655 .

[6] A. Stern, Phys. Rev. Lett. 68 (1992) 1022.

[7] Y. Meir, Y. Gefen and O. Entin-Wohlman, Phys. Rev. Lett. 63 (1989) 798.

[8] V. Chandrasekhar, R.A. Webb, M.J. Brady, M.B. Ketchen, W.J. Gallagher and A. Kleinsasser, Phys. Rev. Lett. 67 (1991) 3578.

[9] D. Mally, C. Chapelier and A. Benoit, Phys. Rev. Lett. 70 (1993) 2020.

[10] Y. Gefen, Y. Imry and M.Ya. Azbel, Phys. Rev. Lett. 52 (1984) 129.

[11] A.A. Abrikosov, Fundamentals of the Theory of Metals (North-Holland, Amsterdam, 1988).
[12] H.F. Cheung, E.K. Riedel and Y. Gefen, Phys. Rev. Lett. 62 (1989) 587

[13] G. Montambeau, H. Bouchiat, D. Siegel and R. Friesner, Phys. Rev. B 42 (1990) 7647.

[14] B.L. Altshuler, Y. Gefen and Y. Imry, Phys. Rev. Lett. 66 (1991) 88.

[15] L.P. Levy, G. Dolan, J. Dunsmuir and H. Bouchiat, Phys. Rev. Lett. 64 (1990) 2074

[16] F. London, Superfluids, Vol.1 (Wiley, New York, 1950).

[17] 1.O. Kulik, A.S. Rozhavsky and E.N. Bogachek, Pis'ma Zh. Eksp. Teor. 47 (1988) 251 [JETP Lett. 47 (1988) 302].

[18] J.F. Weisz, R. Kishore and F.V. Kusmartsev, Phys. Rev. B 49 (1994) 8126

[19] T. Swahn, E.N. Bogachek, Yu.M. Galperin, M. Jonson and R. Shekhter, Phys. Rev. Lett. 73 (1994) 162

[20] S. Washburn, H. Schmid, D. Kern and R.A. Webb, Phys. Rev. Lett. 59 (1987) 1791.

[21] D.V. Averin and K.K. Likharev, Single electronics, in: Mesoscopic Phenomena in Solids, eds. B.L. Altshuler, P.A. Lee and R.A. Webb (North-Holland, Amsterdam, 1991).

[22] I.K. Yanson, Fiz. Nizk. Temp. 9 (1983) 676 [Sov. J. Low Temp. Phys. 9 (1983) 343]

[23] I.O. Kulik, Fiz. Nizk. Temp. 18 (1992) 450 [Sov. J. Low Temp. Phys. 18 (1992) 302].

[24] I.O. Kulik, to be published.

[25] B.K. Ridley in: Negative Differential Resistance and Instabilities in 2-D Semiconductors, eds. N. Balkan, B.K. Ridley and A.J. Vickers (Plenum, New York, 1993) p.1. 